Supporting Information

\title{
High Molecular Weight Polyacrylonitrile Precursor for S@pPAN Composite Cathode Materials with High Specific Capacity for Rechargeable Lithium Batteries
}

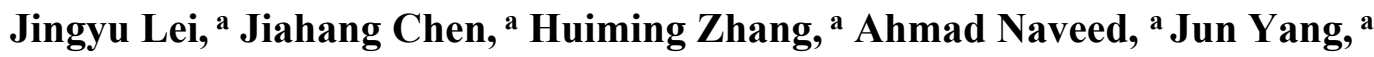 \\ Yanna Nuli, a Jiulin Wangab*
}

a. Department of Chemical Engineering, Shanghai Jiao Tong University, Shanghai 200240, China

b. College of Chemistry, Zhengzhou University, Henan 450001, China

Corresponding author

*Email: wangjiulin@sjtu.edu.cn 
Table S1. Summary of some publications of S@pPAN cathodes for the Li-S battery

\begin{tabular}{cccccc}
\hline Sample name & $\begin{array}{c}\text { Sulfur } \\
\text { Content } \\
(\mathrm{wt} \%)\end{array}$ & $\begin{array}{c}\text { First reversible } \\
\text { specific capacity } \\
(\mathrm{mAh} / \mathrm{g})\end{array}$ & $\begin{array}{c}\text { Capacity retention } \\
(\mathrm{mAh} / \mathrm{g})\end{array}$ & $\begin{array}{c}\text { Cycle } \\
\text { number }\end{array}$ & Ref \\
\hline SPAN & 48 & 672 & $504(0.2 \mathrm{C})$ & 80 & 1 \\
SPAN & 46.2 & 627.9 & $450.5(0.1 \mathrm{C})$ & 80 & 2 \\
MSPAN & 45.87 & 651.4 & $541.3(0.1 \mathrm{C})$ & 30 & 3 \\
SPAN & 45.6 & 649.8 & $456(0.4 \mathrm{C})$ & 100 & 4 \\
SPAN/MWCNT & 40 & 548 & $500(0.2 \mathrm{C})$ & 260 & 5 \\
S/rSP@SPAN & 54.5 & 787.5 & $681.8(0.1 \mathrm{C})$ & 100 & 6 \\
SC-PAN & 44.7 & 618.2 & $559.6(0.1 \mathrm{C})$ & 150 & 7 \\
S@pPAN555 & 54.93 & 798.1 & $733.9(0.2 \mathrm{C}, \mathrm{RT})$ & 200 & This work \\
S@pPAN555 & 54.93 & $900.9\left(0.1 \mathrm{C}, 50^{\circ} \mathrm{C}\right)$ & $797.6\left(0.5,50^{\circ} \mathrm{C}\right)$ & 200 & This work \\
\hline
\end{tabular}



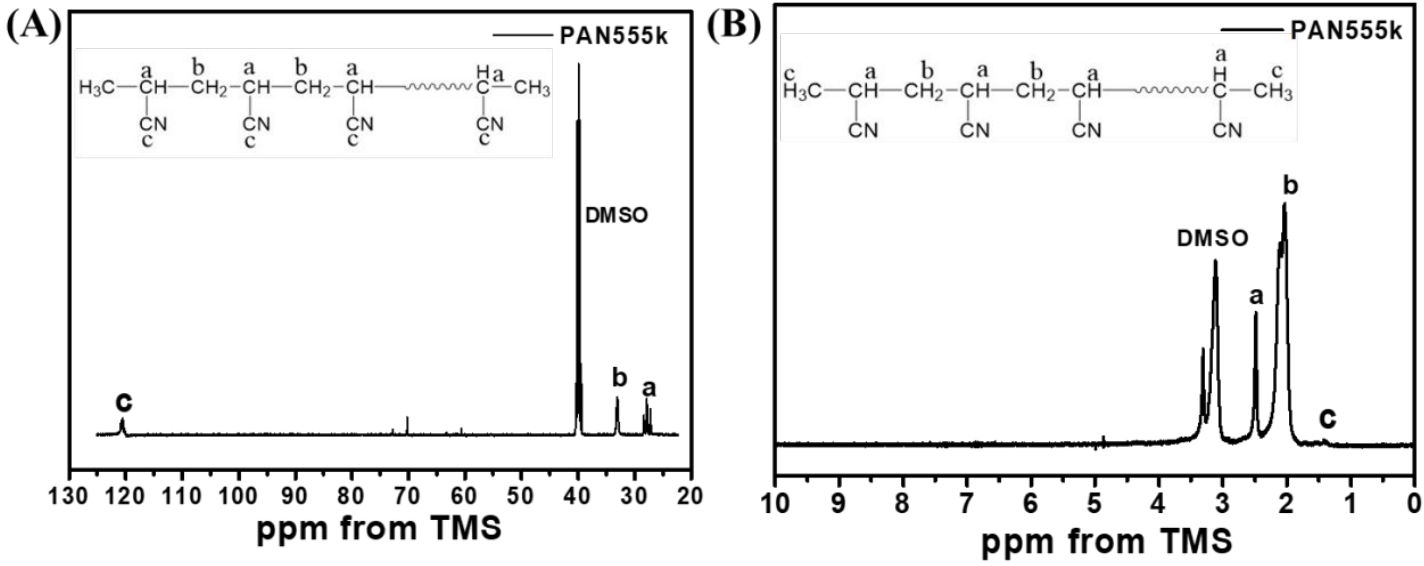

Figure S1. ${ }^{13} \mathrm{C}(\mathrm{A})$ and ${ }^{3} \mathrm{H}$ NMR(B) of PAN555k precursor 


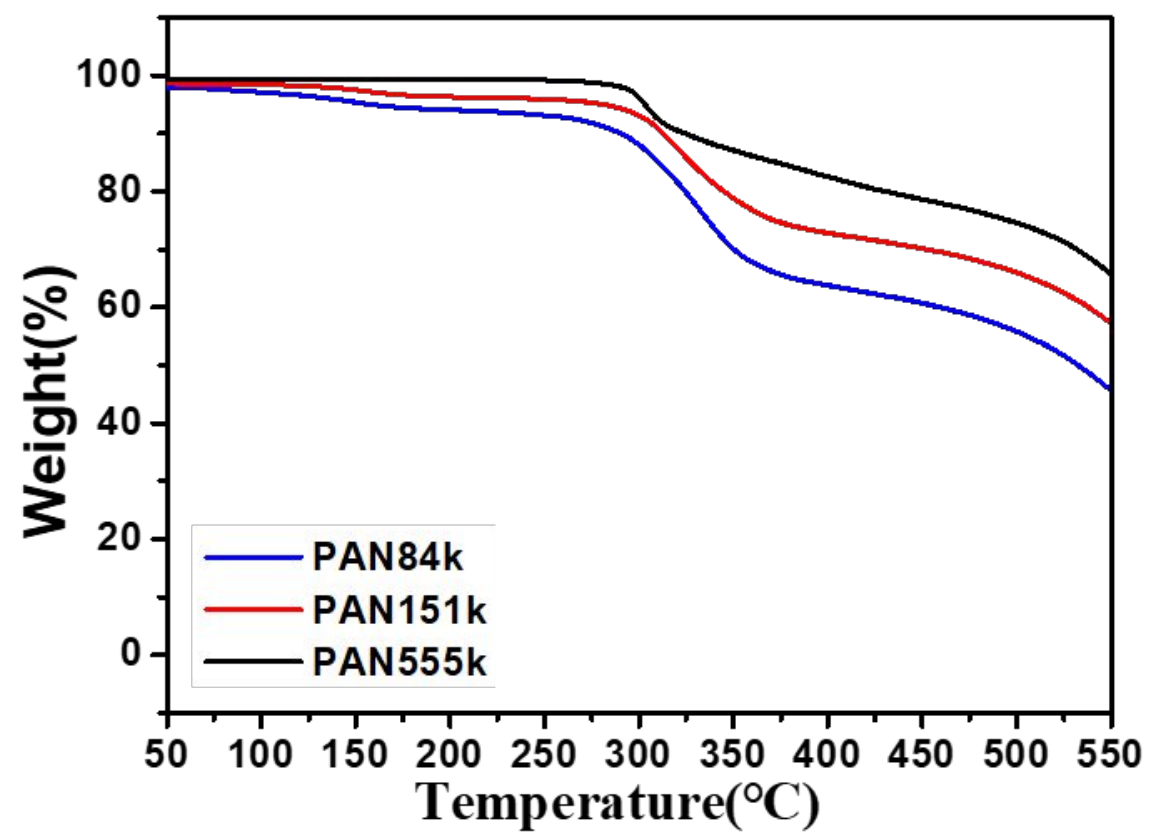

Figure S2. TGA curves of PAN precursors (where the number following PAN indicates its molecular weight). 


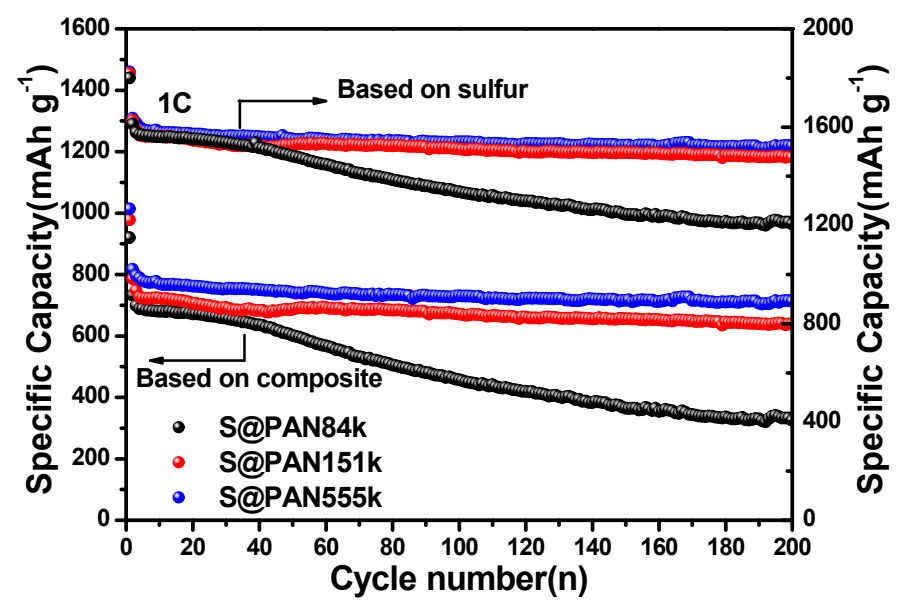

Figure S3. Cycling performance of S@pPAN at 1C (where the number following PAN indicates its molecular weight). 
Scheme S1. The schematic of S@pPAN84k;S@pPAN151k and S@pPAN555k
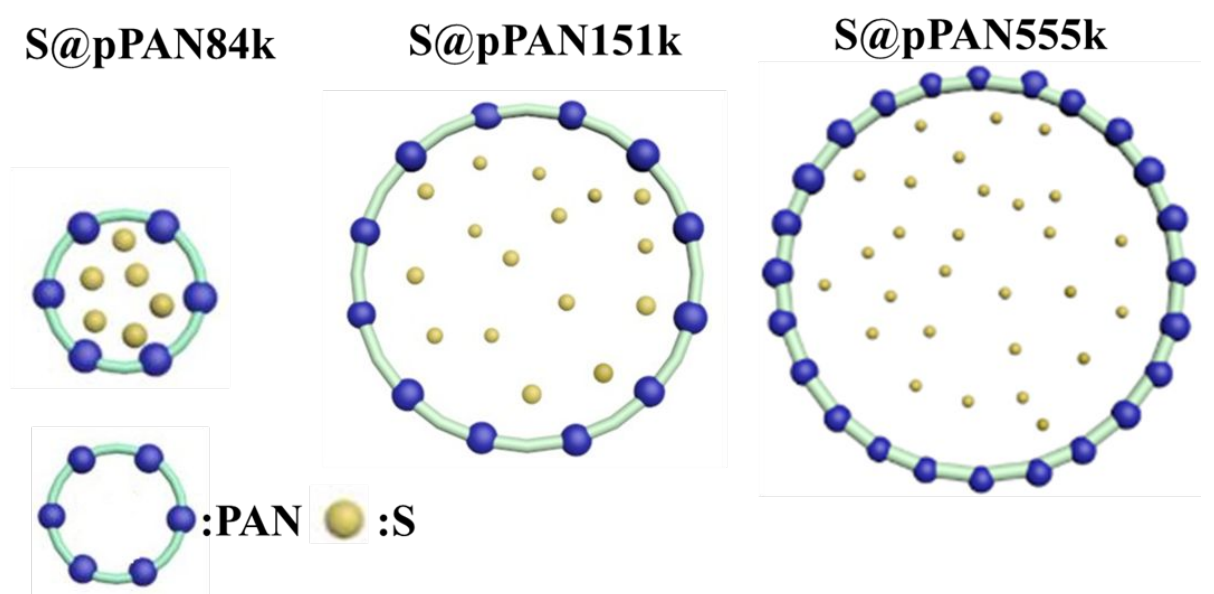


\section{Reference}

(1) Konarov, A.; Gosselink, D.; Doan, T. N. L.; Zhang, Y.; Zhao, Y.; Chen, P., Simple, Scalable, and Economical Preparation of Sulfur-PAN Composite Cathodes for Li/S Batteries. J. Power Sources 2014, 259, 183-187.

(2) Li, J.; Li, K.; Li, M.; Gosselink, D.; Zhang, Y.; Chen, P., A Sulfur-Polyacrylonitrile/Graphene Composite Cathode for Lithium Batteries with Excellent Cyclability. J. Power Sources 2014, 252, 107-112.

(3) Liu, Y.; Haridas, A. K.; Cho, K.-K.; Lee, Y.; Ahn, J.-H., Highly Ordered Mesoporous Sulfurized Polyacrylonitrile Cathode Material for High-Rate Lithium Sulfur Batteries. J. Phys. Chem. C 2017, 121 (47), 26172-26179.

(4) Wei, S.; Ma, L.; Hendrickson, K. E.; Tu, Z.; Archer, L. A., Metal-Sulfur Battery Cathodes Based on PAN-Sulfur Composites. J. Am. Chem.Soc. 2015, 137 (37), 12143-12152.

(5) Mentbayeva, A.; Belgibayeva, A.; Umirov, N.; Zhang, Y.; Taniguchi, I.; Kurmanbayeva, I.; Bakenov, Z., High Performance Freestanding Composite Cathode for Lithium-Sulfur Batteries. Electrochem. Acta 2016, 217, 242-248.

(6) Weret, M. A., Sulfurized-Poly (acrylonitrile) Wrapped Carbonsulfur Composite Cathode Material for High Performance Rechargeable Lithium-Sulfur Batteries. J. Power Sources 2019, 412, 670-676.

(7) Wang, X.; Qian, Y.; Wang, L.; Yang, H.; Li, H.; Zhao, Y.; Liu, T., Sulfurized Polyacrylonitrile Cathodes with High Compatibility in Both Ether and Carbonate Electrolytes for Ultrastable Lithium-Sulfur Batteries. Adv. Funct. Mater. 2019, 29 (39), 1902929. 